\title{
Discriminating signs: Perceptual precursors to acquiring a visual-gestural language
}

\author{
Makeba Parramore Wilbourn*, Marianella Casasola \\ Cornell University, Department of Human Development, MVR Hall, Ithaca, \\ NY 14853, United States
}

Received 6 April 2006; received in revised form 8 August 2006; accepted 11 August 2006

\begin{abstract}
We tested hearing 6- and 10-month-olds' ability to discriminate among three American Sign Language (ASL) parameters (location, handshape, and movement) as well as a grammatical marker (facial expression). ASL-naïve infants were habituated to a signer articulating a two-handed symmetrical sign in neutral space. During test, infants viewed novel two-handed signs that varied in only one parameter or in facial expression. Infants detected changes in the signer's facial expression and in the location of the sign but provided no evidence of detecting the changes in handshape or movement. These findings are consistent with children's production errors in ASL and reveal that infants can distinguish among some parameters of ASL more easily than others.
\end{abstract}

(C) 2006 Elsevier Inc. All rights reserved.

Keywords: Perception; Discrimination; American Sign Language; Parameters; Infants

Infants possess remarkable perceptual abilities that are pivotal in the acquisition of language. For hearing infants, the ability to discriminate between speech contrasts plays an essential role in the acquisition of spoken language. Until about 6 months of age, infants are able to discriminate between speech contrasts from non-native languages (e.g., / $/ t^{\prime}$ a/ versus /ta/ in Hindi) and their native language (e.g., /ba/ versus /da/ in English; Werker \& Tees, 1984). Thus, infants' perceptual abilities, early in their first year, allow for "language-universal phonetic discrimination", even though by 12 months, infants' ability to discriminate between non-native speech contrasts begins to decline (Werker \& Tees, 1999 , p. 515). These findings raise the question of whether infants' perceptual abilities play an analogous role in the acquisition of a visual-gestural language. To date, we know substantially less about hearing infants' sensitivity to and discrimination of the linguistic properties of a visual-gestural language, such as American Sign Language (ASL). The purpose of the present study is to explore how hearing infants' perceptual sensitivities to the linguistic properties of ASL may aid them in acquiring a visual-gestural language.

The linguistic properties of visual-gestural languages, such as ASL, are notably similar to the phonological structure of spoken languages. Stokoe (1960) and Stokoe, Casterline, and Croneberg (1965) outlined three primary parameters ${ }^{1}$ that distinguish signs: (1) the location where the sign is articulated; (2) the shape of the hand(s) or handshape; and

\footnotetext{
* Corresponding author. Tel.: +1 607254 1388; fax: +1 6072559856.

E-mail address: mmp26@cornell.edu (M.P. Wilbourn).

${ }^{1}$ Stokoe (1960) and Stokoe et al. (1965) also included palm orientation, the direction of the palm in relation to the signer's body (e.g., palm out, palm down) as a parameter of ASL, however in the current study this distinction was not directly examined and was held constant in each of the sign presentations (i.e., palm out and palm in).
} 
(3) the movement of the hand(s). Also similar to a spoken language, ASL has minimal pairs of signs (i.e., contrasts) that differ in only one parameter. For instance, the signs $<$ SUMMER $>$ and $<$ UGLY $>$ vary with respect to location but maintain the same handshape and movement (Klima \& Bellugi, 1979). Although manually articulated, these parameters serve the same essential function as phonemes in spoken languages.

Likewise, the use of facial expressions in ASL serves a similar function to intonation in English; particularly, in the contrasts that denote yes/no questions from wh-questions. In English, yes/no questions are typically pronounced with a rising intonation whereas wh-questions are pronounced with a falling intonation, a distinction that is essential for effective communication (Frishberg, 1975). Similarly in ASL, yes/no questions are articulated with raised eyebrows whereas wh-questions are articulated with a furrowed brow (Sternberg, 1998). This distinction is critical, grammatically, in that questions missing the appropriate facial expression can be completely uninterpretable (Campbell, Woll, Benson, \& Wallace, 1999).

The similarities between English and ASL raise the question of what role hearing infants' perceptual abilities plays in their acquisition of a visual-gestural language. Just as infants' acute perceptual sensitivities form the necessary building blocks to acquire a spoken language, it seems plausible that these sensitivities serve an analogous function in the acquisition of a visual-gestural language, such as ASL (Holmes \& Holmes, 1980; Prinz \& Prinz, 1979). There have been only a few studies that have explored this issue by examining hearing infants' ability to discriminate ASL contrasts (Carroll \& Gibson, 1986; Schley, 1991). Schley (1991), for example, examined ASL-naïve hearing 3.5-month-olds' discrimination of two different types of movement contrasts in ASL. Infants were habituated to one particular type of movement (e.g., cyclicity) and tested with signs that had a different type of movement (e.g., direction). She found that ASL-naïve hearing infants were able to discriminate between various movement contrasts in ASL. Similarly, Carroll and Gibson (1986) found that hearing 4-month-olds were able to discriminate between two ASL signs based on multiple movement dimensions $(<\mathrm{TEN}>$ versus $<$ OTHER $>$ ) as well as on a single movement dimension $(<$ LEFT $>$ versus $<$ LIBRARY $>$ ). Although these studies are some of the only investigations into hearing infants' sensitivities to visual-gestural languages (see also Masataka, 1995), the findings do not address hearing infants' ability to discriminate among the other ASL parameters, such as handshape and location. Do hearing infants discriminate between these parameters equally well?

Several lines of research with adults and children suggest differences in how easily particular parameters of ASL are discriminated, even for experienced signers. For instance, adult native signers demonstrate more difficulty in discriminating and identifying changes in handshape (Stungis, 1981) and movement (Tartter \& Fischer, 1982) than changes in location (Poizner \& Lane, 1978). Likewise, 6 to 10-year-old Deaf children made more errors discriminating minimal pairs of signs that differed in handshape than in movement or location (Hamilton, 1986). These findings suggest that changes in handshape and movement are more difficult to discriminate than changes in location. Perhaps, novice ASL learners, such as hearing infants, may demonstrate the same pattern of difficulty as experienced ASL signers.

The suggestion that changes in handshape and movement may be more difficult to discriminate between than changes in location is also supported by previous research examining young signers' production errors during the early stages of ASL acquisition. For instance, research on the ASL production errors of Deaf and hearing infants and toddlers demonstrates that first signs are typically produced in the correct location but with incorrect handshapes and movement (Bonvillian \& Siedlecki, 2000; Wilbour \& Jones, 1974). Although these errors have been attributed primarily to motoric constraints (Conlin, Mirus, Mauk, \& Meier, 2000), Bonvillian and Siedlecki (2000) and Siedlecki and Bonvillian (1993) argue that these errors also may be due to difficulty in perceiving specific handshapes. Thus, perceptual abilities may also play a role in children's production errors in ASL.

Bearing in mind that the ability to discriminate among the primary parameters of ASL plays an essential role in the acquisition of ASL, the current study sought to outline the degree to which hearing infants' perceptual abilities provide the scaffolding needed to acquire this type of visual-gestural language. Specifically, we tested hearing ASLnaïve infants' ability to discriminate among three primary parameters (location, handshape, movement) as well as a grammatical marker (facial expression) in ASL. Six- and 10-month-olds were tested in order to explore possible developmental changes in these abilities. The infants were habituated to a single sign (i.e., a two-handed symmetrical sign) and tested with novel signs that varied in only one parameter (i.e., handshape, movement, location, or facial expression). Two-handed symmetrical signs (both hands mirroring one another) were used because they are representative of the first signs children typically produce as well as signs typically articulated in neutral space (Frishberg, 1975). 


\section{Method}

\subsection{Participants}

Participants were recruited at a local hospital at the time of their birth. At that time, parents were given a letter and subsequently contacted to participate. Fifteen 6-month-olds ( \pm 2 weeks, 7 females) and twelve 10-month-olds ( \pm 2 weeks, 6 females) participated. Infants were full-term, of normal birth weight, and had no auditory or visual problems. Infants who had been previously exposed to ASL or "baby signs" were excluded. Six additional infants were excluded (one 6-month-old and five 10-month-olds) because they failed to meet the habituation criterion (described below). Four additional infants (two at each age) were excluded because they demonstrated extreme looking times $(+2 S D)$ to the familiar event.

\subsection{Stimuli}

Fig. 1 depicts representative examples of the stimuli. ${ }^{2}$ The stimuli were videotaped dynamic events that depicted a female signer producing a two-handed symmetrical sign in front of her torso (i.e., neutral space). The habituation event depicted the signer slowly lifting her hands up from her lap and producing the ASL sign, $<$ FINISH $>$. This sign was produced twice in rapid succession to ensure that infants had sufficient exposure to the sign. As she produced the sign, the signer raised her eyebrows which is a grammatical marker in ASL used to indicate a yes/no question.

Test events were created that depicted novel signs in which one parameter differed from the habituation sign. More specifically, these novel signs depicted a change in handshape, location or movement while maintaining the other parameters in the habituation sign. Each novel sign was based on an actual ASL sign but was modified slightly to ensure that only the relevant parameter differed from the habituation sign. In the novel handshape event, the signer's hands changed from all of the fingers being extended (open 5-hand) to where only the thumb and pinky finger were extended (Y-hand) (see Fig. 1). This change yielded a sign that resembled the ASL sign for $\langle$ PLAY $\rangle$, yet differed slightly in that the Y-hands faced out instead of down (Sternberg, 1998). Because the ASL sign for $<$ PLAY $>$ presented a change in more than one parameter (i.e., handshape and palm orientation), this modified version was used instead.

The novel location event depicted the sign $<$ FINISH $>$ produced above the signer's forehead instead of in neutral space. This sign resembled the ASL sign for $<$ GREAT $>$. However, this modified sign differed in movement slightly from the actual ASL sign for $<$ GREAT $>$, which is typically articulated by having the hands move up and out (Sternberg, 1998). The novel movement event depicted a change in the rotating-type movement used in the $<$ FINISH $>$ sign to, instead, having the hands in closed fists, and then quickly opening and extending the fingers, similar to the ASL sign, $<$ MANY $>$ (Sternberg, 1998). For the novel facial expression event (hereafter, novel expression), the only change was that the signer presented $<$ FINISH $>$ with a furrowed brow rather than raised eyebrows.

In order to determine the extent to which the modified signs varied from the actual ASL signs, four Deaf native signers rated the modified ASL signs on a 1 (unrecognizable) to 5 (native-like) scale. The habituation sign $<$ FINISH $>$ received an average rating of 4.6 , the novel movement sign, $<$ MANY $>$ received an average rating of 4.4 , and the novel handshape sign, $<\mathrm{PLAY}>$ and novel location sign, $<\mathrm{GREAT}>$ received average ratings of 2.3. Hence, $<$ FINISH $>$ and $<$ MANY $>$ were more native-like in their articulation, whereas $<$ PLAY $>$ and $<$ GREAT $>$ were sufficiently modified so that they only resembled the actual ASL signs. Although these modifications reduced the ecological validity of the ASL signs, they allowed us to increase the internal validity to pinpoint the basis of infants' discrimination of each parameter.

To address whether some parameters are more distinct on a psychophysical basis, we documented ASL-naïve hearing adults' discrimination of the signs. We asked 10 hearing adults, who were naïve to any visual-gestural language, to compare each of the novel signs to the habituation sign $<$ FINISH $>$. Adults rated the similarity of each novel sign to $<$ FINISH $>$ on a scale of 1 (highly similar to $<$ FINISH $>$ ) to 5 (low similarity to $<$ FINISH $>$ ). Adults rated the sign $<$ MANY $>$ (i.e., the change in movement) as most different $(M=4.1, S D=.71)$, but also rated the sign $<$ GREAT $>$ (i.e., the change in location, $M=3.3, S D=1.74$ ) and the sign $<$ PLAY $>$ (i.e., the change in handshape, $M=3.1, S D=.86$ ) as being fairly different from $<$ FINISH $>$. In contrast, the raised versus furrowed eyebrows (i.e., the change in facial

\footnotetext{
${ }^{2}$ Representative examples of the stimuli are presented because the signer did not grant her permission for us reproduce her videotaped image, thus we have used a different signer to exemplify the videotaped dynamic events.
} 
Example of Habituation and Familiar Test Event - ASL Sign <FINISH $>$
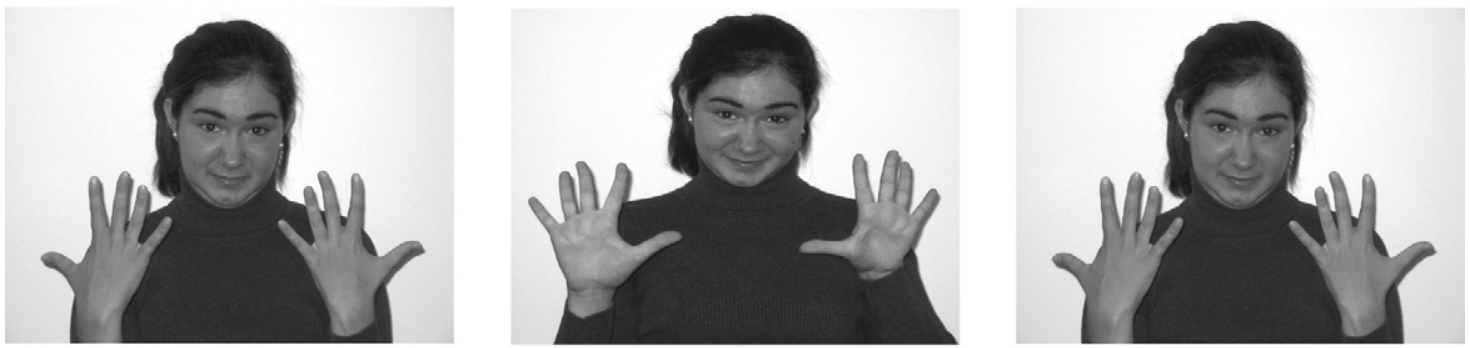

Example of Novel Handshape Test Event - ASL Sign <PLAY>
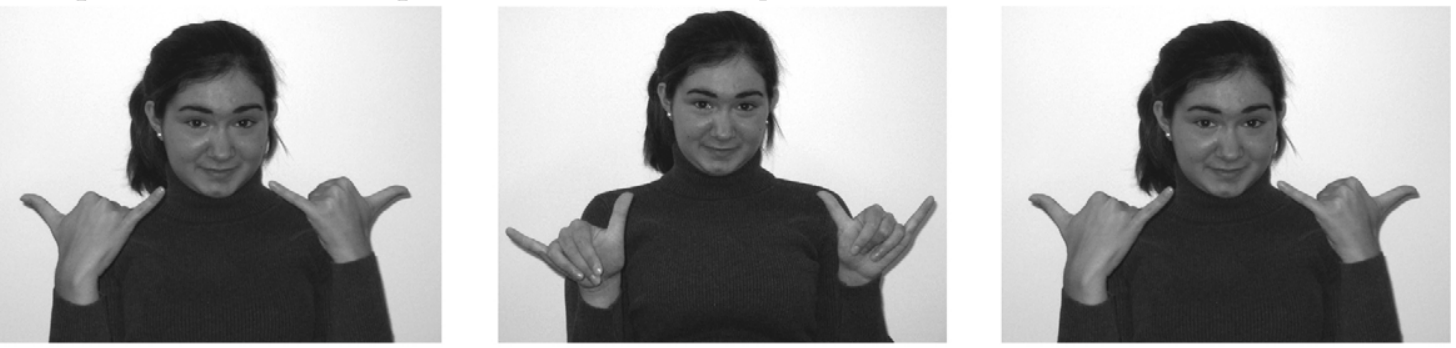

Example of Novel Movement Test Event - ASL Sign $<$ MANY $>$
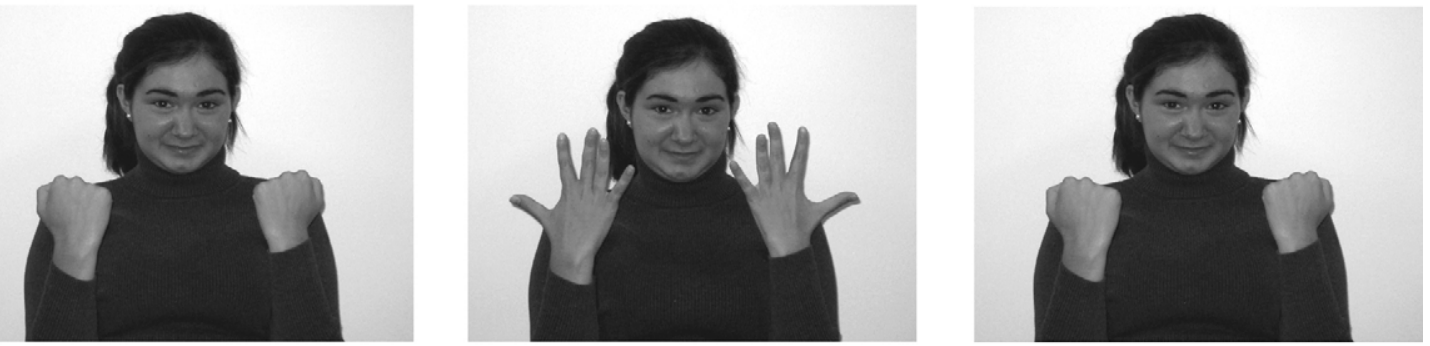

\section{Example of Novel Location Test Event - ASL Sign <GREAT $>$}
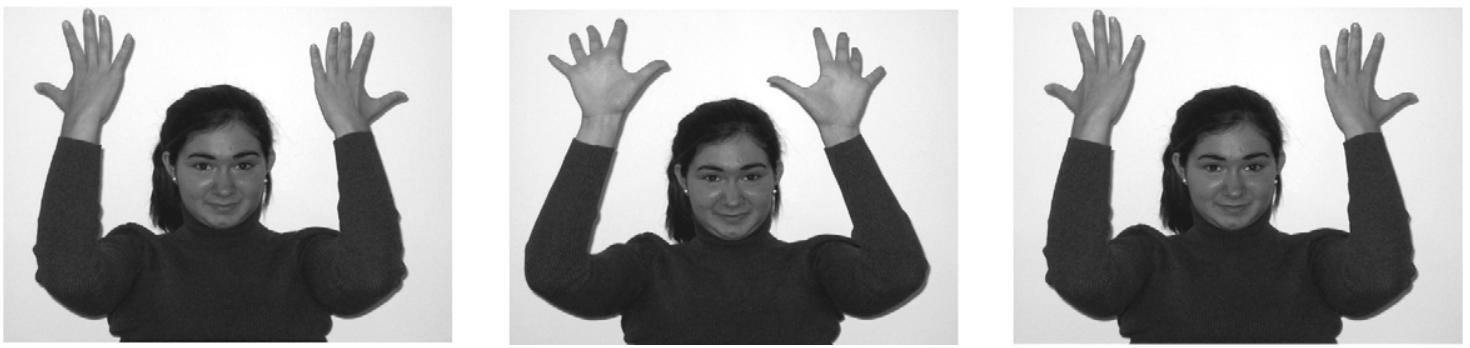

Fig. 1. Representative examples of the 2-handed signs presented in the dynamic events.

expression) were rated as highly similar $(M=1.3, S D=.48)$. Therefore, adults viewed the change in movement as the most different, the change in location and handshape to be equally different, and considered the change of expression to be the least salient change.

For both the habituation and test events, the signs were produced twice rapidly with a $1 / 2$-second pause between each presentation before the signer returned her hands to her lap. This double presentation was looped 10 times without pauses to create a $30 \mathrm{~s}$ trial. 


\subsection{Apparatus}

Infants were seated on their parent's lap approximately $127 \mathrm{~cm}$ from a 20 -inch computer monitor. Below this monitor was a camera that was connected to another monitor and VCR in an adjoining room, where an experimenter observed and recorded the infants' looking times during the habituation and test trials. The presentation and recording of each trial was done using G5 Macintosh computer and Habit 2000 software (Cohen, Atkinson, \& Chaput, 2000).

\subsection{Procedure}

After parental consent was obtained, the infant and his or her parent were seated in the testing room. Parents were asked to remain silent and neutral during the testing session. From the adjoining room, an experimenter depressed a key on the computer to begin the testing session. Prior to each trial, an attention-getter (a green chiming expanding circle) was used to focus the infant's attention. Once the infant's attention was focused on the monitor, the experimenter depressed a key to begin a trial. To record looking time, the experimenter held down a key for as long as the infant attended to the trial. A trial played until the infant either looked away for more than $1 \mathrm{~s}$ or until the $30 \mathrm{~s}$ trial ended.

\subsection{Pretest \& habituation phase}

The pretest trial (a hand moving a stuffed pig) was presented first to introduce the procedure. After the pretest, the habituation phase began. During habituation, infants viewed the signer producing the sign $<$ FINISH $>$. The habituation criterion was met when an infant's looking time across three habituation trials decreased by $50 \%$ from the first three habituation trials. Habituation trials ended when this criterion was met or when 20 habituation trials had been presented.

\subsection{Test phase}

Following habituation, infants viewed five test trials. One familiar trial presented the same event seen during habituation (i.e., the sign <FINISH $>$ ), and four trials presented a novel handshape, a novel movement, a novel location, or a novel expression. The presentation order of the five test trials was counterbalanced across participants. An independent observer coded the looking times of eight randomly chosen infants from the recorded sessions, yielding an inter-rater reliability of .996 (range $=.989$ to .999$)$.

\section{Results}

To ensure that infants did not meet the habituation criterion as an artifact, an analysis was conducted comparing infants' average looking time during the first three habituation trials to the familiar test event (i.e., habituation event). A 2 (Age: 6 months, 10 months) $\times 2$ (Sex: males, females) $\times 2$ (Trials: average of first three habituation trials, familiar test trial) mixed-model analysis of variance (ANOVA) was conducted and revealed a significant main effect of Trials, $F(1,23)=67.21, p<.001, \eta_{p}^{2}=.75$. Infants looked significantly longer at the habituation event during the first three trials of habituation $(M=18.5 \mathrm{~s}, S D=6.7 \mathrm{~s})$ than at the same event when it was presented as a test trial $(M=6.2 \mathrm{~s}$, $S D=3.2 \mathrm{~s}$ ). No other significant main effects or interactions emerged, all $p$ 's $>.05$.

Table 1 presents infants' mean looking times and standard deviations to each event during the test trials. To address whether infants discriminated each novel event from the familiar test event, a 2 (Age) $\times 2($ Sex $) \times 5$ (Test Event: familiar,

Table 1

Infants' mean looking time in seconds with standard deviations to the two-handed sign test events as function of age

\begin{tabular}{lccr}
\hline Test event & 6-month-olds & 10-month-olds & Total sample \\
\hline Familiar & $5.96(3.69)$ & $6.50(2.81)$ & $6.20(3.27)$ \\
Novel handshape & $8.73(7.02)$ & $8.01(3.63)$ & $8.41(5.68)$ \\
Novel movement & $7.60(5.02)$ & $9.33(6.97)$ & $8.37(5.91)$ \\
Novel location & $10.15(6.74)$ & $15.45(10.89)$ & $12.5(9.05)$ \\
Novel expression & $9.19(7.22)$ & $9.76(5.95)$ & $9.44(6.57)$ \\
\hline
\end{tabular}


novel handshape, novel movement, novel location, novel expression) ANOVA was conducted. Simple contrasts revealed that infants looked significantly longer at the novel location event relative to the familiar event, $F(1,23)=11.76, p<.01$, $\eta_{p}^{2}=.34$. That is, infants looked significantly longer at the sign when it was produced above the head rather than in its familiar location in neutral space. Infants also discriminated between the novel expression with the furrowed brow and the familiar expression with the raised eyebrows, $F(1,23)=4.94, p<.05, \eta_{p}^{2}=.18$, although the effect size for this result was small. However, infants did not provide evidence of discriminating between the novel handshape event and the familiar event, $F(1,23)=3.10, p>.05, \eta_{p}^{2}=.12$ nor between the novel movement event and the familiar event, $F(1,23)=2.55, p>.10, \eta_{p}^{2}=.09$. No other significant main effects or interactions emerged, all $p$ 's $>.05$.

A subsequent statistical analysis was conducted to examine the degree to which the changes in location and facial expression were more salient to infants compared to the changes in handshape and movement. For each infant, a difference score was computed (i.e., looking time to the novel test event-looking time to the familiar test event). This difference score was subjected to a $2(\mathrm{Age}) \times 2(\mathrm{Sex}) \times 4$ (Difference Score: handshape, location, movement, expression) mixed-model ANOVA. Simple contrasts comparing the novel location difference score to each of the remaining difference scores revealed that infants looked significantly longer at the change in location relative to the changes in handshape, $F(1,23)=5.96, p<.05, \eta_{p}^{2}=.21$, movement, $F(1,23)=5.65, p<.05, \eta_{p}^{2}=.20$, and expression, $F(1,23)=5.68, p<.05, \eta_{p}^{2}=.20$. No other significant main effects or interactions emerged, all $p$ 's $>.05$.

Interestingly, the simple contrasts comparing the novel expression difference score to each of the remaining difference scores revealed very different results. The infants did look significantly less at the changes in expression relative to the changes in location, $F(1,23)=5.68, p<.05, \eta_{p}^{2}=.20$, but did not look significantly longer to the changes in expression relative to the changes in handshape or movement, both $p$ 's $>.10$. No other significant main effects or interactions emerged, all $p$ 's $>.05$.

\section{Discussion}

The current study demonstrated that when ASL-naïve hearing 6- and 10-month-olds were habituated to a two-handed symmetrical sign and tested with signs that varied in only handshape, location, movement, or facial expression, they detected the change in location and facial expression. Specifically, infants detected the change from a raised brow to a furrowed brow. Infants were especially attentive to the change in the location from the sign $<$ FINISH $>$, articulated in front of the signer's torso, to the sign $<$ GREAT $>$, articulated above the signer's head, a result that yielded a stronger effect size relative to that obtained for the change in facial expression. However, infants did not provide evidence of detecting the changes in handshape (open 5-hand versus Y-hand) or movement ( $<$ FINISH $>$ versus $<$ MANY $>$ ).

The present results are consistent with previous findings on children's early production errors in ASL. For instance, Wilbour and Jones (1974) report that hearing infants exposed to ASL from birth typically produce their first signs in the correct location but with incorrect handshapes and movement. Likewise, Bonvillian and Siedlecki (2000) found that children in their longitudinal study produced signs in the correct location $84 \%$ of time, with the correct movement and handshape, $61 \%$ and $50 \%$ of the time, respectively. Conlin et al. (2000) contend that these early production errors are due to developing motor abilities that constrain children's ability to produce particular types of signs or parameters correctly. However, the findings from the current study provide some support for the suggestion that young children's errors in the production may not be solely a function of their inability to produce certain parameters accurately, but also a function of their ability to perceive certain parameters accurately (Bonvillian \& Siedlecki, 2000). Hence, the current study lends insight into the specific role perceptual abilities (and not just motor abilities) may play in children's acquisition of a visual-gestural language, like ASL.

But why would some parameters be more perceptually distinct than others? Recall that the ASL-naive adults viewed the change in movement as the most different, the changes in location and handshape to be equally different, and considered the change in expression to be the least salient change. Interestingly, these ratings of the perceptual similarity between ASL signs (i.e., parameters) differed from the infants' discrimination of the test events. Infants did not demonstrate that they detected the change in movement, which was the most perceptually distinct parameter for the adults. In addition, infants did discriminate the change in location but did not provide evidence of discriminating the change in handshape whereas the adults indicated that both of these parameters were equally different from the sign $<$ FINISH $>$. Lastly, the infants did discriminate the change in expression whereas the adults rated this change as the least perceptually distinct. Hence, ASL-naïve infants appear to perceive ASL parameters differently than ASL-naïve adults. 
This developmental change suggests that infants are differentially attuned to ASL parameters than adults, reducing the possibility that infants and adults were simply responding to the overall psychophysical features of the parameters.

Another reason that some parameters may be more perceptually distinct than others for the infant may have had to do with the specific signs selected. The rapid "flicking-type" motion (i.e., outward rotation of the wrists and hands) of the sign may have made it more difficult for infants to attend to changes in movement, since there was such a high degree of motion with both hands. The previous studies that have examined infants' sensitivity to ASL movement contrasts used one-handed ASL signs (Carroll \& Gibson, 1986; Schley, 1991) and not two-handed signs as in the current study. Therefore, infants' sensitivity to movement contrasts may vary depending of the number of hands articulating the sign or alternatively, the specific two-handed sign presented, a possibility not tested in the present study. In addition, the changes in location may have been more perceptually salient regardless of the increased motion of the hands, because the degree of change in location from the sign, $<$ FINISH $>$ to the sign $<$ GREAT $>$ was larger (in front of the signer's torso versus up over the signer's head), than the changes in movement or handshape. These explanations remain to be tested with studies that vary not only the number of hands used to articulate the sign, but also in the degree of change in each parameter.

Another possible explanation as to why the novel location sign (i.e., $<$ GREAT $>$ ) was perceptually distinct for infants is that it deviated much more from its corresponding ASL sign compared to the novel movement and novel handshape signs and their corresponding ASL signs (i.e., $<$ MANY $>$ and $<$ PLAY $>$, respectively) as rated by the Deaf native signers. This raises the concern that the infants' discrimination between the habituation sign $<$ FINISH $>$ and the novel location sign, $<$ GREAT $>$ was due to a spurious stimulus difference. Although possible, this explanation is unlikely for the following reasons. First, all of the infants were naïve to ASL, which eliminated the likelihood that they were using as a basis of comparison the difference between the created novel location sign used in the current study and the actual ASL sign $<$ GREAT $>$. Therefore, even though the Deaf native signers rated this created novel location sign as being less native-like compared to the other created signs, the ASL-naïve infants could not have been influenced by this deviation.

Secondly, the ratings from the ASL-naïve hearing adults also suggest that infants' ability to discriminate the change in location was not due to a spurious stimulus difference. Recall that the hearing adults rated this novel location sign as equally different as the novel handshape sign compared to the familiar sign <FINISH $>$. Thus, if the ASL-naïve infants viewed these events in a similar manner as the hearing ASL-naïve adults then they should have also discriminated the change in handshape, which they did not. In sum, the hearing ASL-naïve infants' discrimination of the ASL parameter of location and grammatical marker of facial expression contrasts with the ratings of both the Deaf native ASL signers and ASL-naïve hearing adults, which demonstrates that infants' initial perception of ASL parameters is quite different than that of adults, who do and do not have experience with a visual-gestural language.

The current finding that infants were sensitive to the ASL contrasts articulated on the face (raised versus furrowed brow) is not surprising in that faces, in general, are salient to young infants. Research has demonstrated that infants, as young as 23 hours, are able to discriminate between various facial expressions (Walker-Andrews, 1997). Thus, not only are hearing infants able to use their perceptual abilities in order to acquire their ambient language, but also are able to use these abilities to detect changes in ASL contrasts articulated on the face, a grammatical distinction not used in English (Reilly, McIntire, \& Bellugi, 1991). However, it is important to consider that the effect size for this result was small and that infants did not look significantly longer at this change compared to the changes in handshape or movement, demonstrating that this change was not as salient for the infants as the change in location.

The findings from the current project underscore that infants were able to recruit their perceptual abilities to detect changes in certain ASL parameters. This ability provides the building blocks necessary to acquire language, whether that language is visual-gestural or spoken. Since the infants in the current study were unfamiliar with ASL, it seems unlikely that they viewed these signs as linguistic, suggesting that infants may have used a more general perceptual mechanism rather than a mechanism specific to language. Finally, there was no evidence to show developmental change between 6 and 10 months in infants' ability to discriminate among the non-native ASL contrasts. Both the 6- and the 10-month-olds demonstrated sensitivity to the parameter change of location and grammatical contrasts on the face.

Given that modified rather than actual signs were used, can these findings be used as an indicator of infants' ability to discriminate between the primary parameters of ASL? Increasing the internal validity led to a decrease in the ecological validity of the signs. Nonetheless, the modification of the signs made it possible to assess infants' discrimination of the specific changes in handshape, location, or movement. However, the use of modified signs may have made the task slightly more difficult for infants in that the presentation of the signs was less naturalistic. On the other hand, if hearing infants are able to discriminate changes in ASL parameters with modified signs, then it is plausible that they can do 
so with unmodified ASL signs as well. Nevertheless, a task for future research is to document the degree to which the current results are replicable with more naturalistic signs.

\section{Acknowledgments}

This research was supported by a fellowship from the National Academy of Sciences Ford Foundation awarded to Makeba Parramore Wilbourn and grants from the National Science Foundation (PECASE - BSC-0349183) and the National Institute of Health (R03-HD-43941-04) awarded to Marianella Casasola. We wish to express our sincere appreciation of Amanda Purington, Greggy Laroche, Cory Sinclair, Erica Roizen, Geunwon Kim, and Maggie Mirch for their assistance in data collection as well as Ariel Fuch and Anna Hays for their assistance in creating the representative examples of the dynamic events. We would like to sincerely thank Steve Robertson, Jui Bhagwat, the HD writing group, and an anonymous reviewer for their helpful suggestions on previous versions of this manuscript. We would also like to acknowledge the Boynton Middle School Homework Club, Jim Meyers, Michelle Hochstettler as well as the parents and infants that participated in study.

\section{References}

Bonvillian, J. D., \& Siedlecki, T. (2000). Young children's acquisition of the formational aspects of American sign language: Parental report findings. Sign Language Studies, 1, 45-64.

Campbell, R., Woll, B., Benson, P. J., \& Wallace, S. B. (1999). Categorical perception of face actions: Their role in sign language and in communicative face displays. Quarterly Journal of Experimental Psychology, 52A, 62-95.

Carroll, J. J., \& Gibson, E. J. (1986). Infant perception of gestural contrasts: Prerequisites for the acquisition of a visually specified language. Journal of Child Language, 13, 31-49.

Cohen, L. B., Atkinson, D. J., \& Chaput, H. H. (2000). Habit 2000: A new program for testing infant perception and cognition [Computer software]. Austin: The University of Texas.

Conlin, K. E., Mirus, G. R., Mauk, C., \& Meier, R. P. (2000). The acquisition of first signs: Place, handshape, and movement. In C. Chamberlain, J. P. Morford, \& R. I. Mayberry (Eds.), Language acquisition by eye (pp. 51-69). Mahwah, N.J.: Lawrence Erlbaum Associates.

Frishberg, N. (1975). Arbitrariness and iconicity: Historical change in American sign language. Language, 51, 696-719.

Hamilton, H. (1986). Perception of sign features by deaf children. Sign Language Studies, 50, 73-77.

Holmes, K. M., \& Holmes, D. W. (1980). Signed and spoken language development in a hearing child of hearing parents. Sign Language Studies, 28, 239-254.

Klima, E. S., \& Bellugi, U. (1979). The signs of language. Cambridge, Mass: Harvard University Press.

Masataka, N. (1995). Perception of motherese in Japanese sign language by 6-month-old hearing infants. Developmental Psychology, 34, 241-246.

Poizner, H., \& Lane, H. (1978). Discrimination of location in American sign language. In P. Siple (Ed.), Understanding language through sign language research (pp. 271-287). New York: Academic Press.

Prinz, P. M., \& Prinz, E. A. (1979). Simultaneous acquisition of ASL and spoken English: Phase I, early lexical development. Sign Language Studies, 25, 283-296.

Reilly, J. S., McIntire, M. L., \& Bellugi, U. (1991). Baby face. A new perspective on universals in language acquisition. In P. Siple, \& S. D. Fischer (Eds.), Theoretical issues in sign language research (pp. 9-23). Chicago, IL: University of Chicago Press.

Schley, S. (1991). Infant discrimination of gestural classes: Precursors of ASL acquisition. Sign Language Studies, 72, $277-296$.

Siedlecki, T., Jr., \& Bonvillian, J. (1993). Location, handshape, and movement: Young children's acquisition of the formational parameters of American sign language. Sign Language Studies, 78, 31-52.

Sternberg, M. L. A. (1998). American sign language dictionary (3rd ed.). New York: Harper Perennial.

Stokoe, W. C. (1960). Sign language structure: An outline of the visual communication system of the American deaf. Buffalo: Department of Anthropology and Linguistics, University of Buffalo.

Stokoe, W. C., Casterline, D. C., \& Croneberg, C. G. (1965). A dictionary of American sign language on linguistic principles. Washington, D.C.: Gallaudet College Press.

Stungis, J. (1981). Identification and discrimination of handshape in American sign language. Perception and Psychophysics, 29, $261-276$.

Tartter, V. C., \& Fischer, S. D. (1982). Perceiving minimal distinctions in ASL under normal and point-light display conditions. Perception and Psychophysics, 32, 327-334.

Walker-Andrews, A. S. (1997). Infants' perception of expressive behaviors: Differentiation of multimodal information. Psychological Bulletin, 121, 437-456.

Werker, J. F., \& Tees, R. C. (1984). Cross-language speech perception: Evidence for perceptual reorganization during the first year of life. Infant Behavior and Development, 7, 49-63.

Werker, J. F., \& Tees, R. C. (1999). Influences on infant speech processing: Toward a new synthesis. Annual Review of Psychology, 50, 509-535.

Wilbour, R. B., \& Jones, M. L. (1974). Some aspects of the acquisition of sign language and English by three hearing children of deaf parents. In M. W. LaGaly, R. A. Fox, \& A. Bruck (Eds.), Papers from the tenth regional meeting. Chicago, IL: Chicago Linguistic Society. 